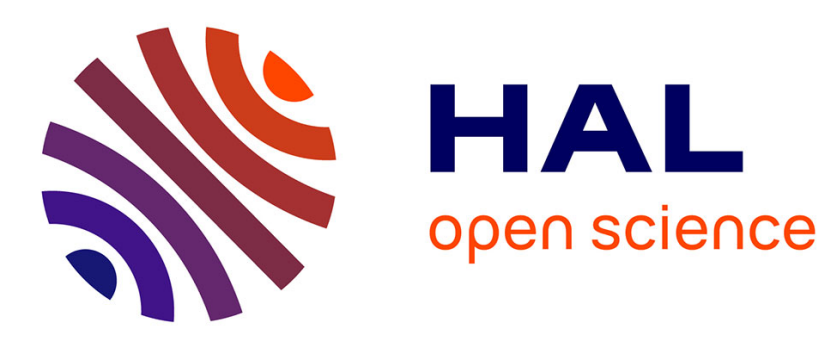

\title{
Social-Spider Optimization Neural Networks for Microwave Filters Modeling
}

\author{
Erredir Chahrazad, Emir Bouarroudj, Mohamed Lahdi Riabi
}

\section{To cite this version:}

Erredir Chahrazad, Emir Bouarroudj, Mohamed Lahdi Riabi. Social-Spider Optimization Neural Networks for Microwave Filters Modeling. 6th IFIP International Conference on Computational Intelligence and Its Applications (CIIA), May 2018, Oran, Algeria. pp.364-372, 10.1007/978-3-31989743-1_32. hal-01913878

\section{HAL Id: hal-01913878 \\ https://hal.inria.fr/hal-01913878}

Submitted on 6 Nov 2018

HAL is a multi-disciplinary open access archive for the deposit and dissemination of scientific research documents, whether they are published or not. The documents may come from teaching and research institutions in France or abroad, or from public or private research centers.
L'archive ouverte pluridisciplinaire HAL, est destinée au dépôt et à la diffusion de documents scientifiques de niveau recherche, publiés ou non, émanant des établissements d'enseignement et de recherche français ou étrangers, des laboratoires publics ou privés. 


\title{
Social-Spider Optimization Neural Networks for Microwave Filters Modeling
}

\author{
Erredir Chahrazad, Emir Bouarroudj and Mohamed Lahdi Riabi \\ Laboratory of electromagnetic and telecommunication, University Brothers Mentouri \\ Constantine1,Constantine,Algeria, \\ cerredir@yahoo.fr
}

\begin{abstract}
In this paper, Social-Spider optimization (SSO) algorithm is proposed for training artificial neural networks (ANN). Further, the trained networks are tested on two microwave filters modeling (Broad-band E-plane filters with improved stop-band and H-plane waveguide filters considering rounded corners). To validate the effectiveness of this proposed strategy, we compared the results of convergence and modeling obtained with the results obtained by NN used a population based algorithm namely Particle Swarm Optimization (PSO-NN). The results prove that the proposed SSO-NN method has given better results.
\end{abstract}

Keywords: Neural Networks, Social-Spider Optimization, Microwave Structures, Modeling.

\section{Introduction}

Microwave passive filters are essential components in the implementation of telecommunications systems. Their main purpose is to pass selected signal and attenuate unwanted signals. Thus, it can clean the communication network by letting only the system band signals to be transmitted or received.

The most popular method for modeling these kinds of filters is the mode matching method (MMM). The mode matching method is often used to solve boundary-value problems involving waveguides. The method consists of decomposing a complex geometrical structure into many regions of simple geometrical form. Hence, in each region, we can find a set of modal functions (or, modes) that satisfy the Maxwell's equations except at the junctions. The problem is that the expanding all the modes in each region with unknown modal coefficients and solving for these coefficients by applying the boundary conditions at the junction of each region, thus making complex mode matching method.

In the past several years, Artificial Neural Network (ANN) provides fast and accurate models for the modeling, simulation, and optimization of microwave component [1-3]. In this paper, we propose a waveguide Filters (Broad-band E-plane filters with improved stop-band and H-plane waveguide filters considering rounded corners) modeling using a multilayer perceptron neural network (MLP-NN) to three layers 
trained by a recently proposed algorithm called Social-Spiders Optimization(SSO) [4]. Each sub-net in the NN architecture shown in Fig.1 , possesses Ne inputs neurons in accordance with the number of the system geometry parameter of structure, Nc neurons in the hidden layer and one output associated with the value of reflection coefficient $\mathrm{S}_{11}\left(f_{\mathrm{k}}\right)$. The entire network consists of $k$ distinct NNs corresponding to a particular frequency with $k$ determined by the number of points in the frequency interval. Frequency responses of $S_{11}$ parameters obtained in simulations compose the network database. The connection weight from the neurons of input layer to the neurons of hidden layer is $W E$ and the connection weight from the neurons of hidden layer to the neurons of output layer is WS.

The paper is organized as follows. Section 2 describes the social-spiders optimization. Training of neural networks using social-spiders optimization is developed in Section 3. Application examples and results are presented in Section 4 and finally, Section 5 makes conclusions.

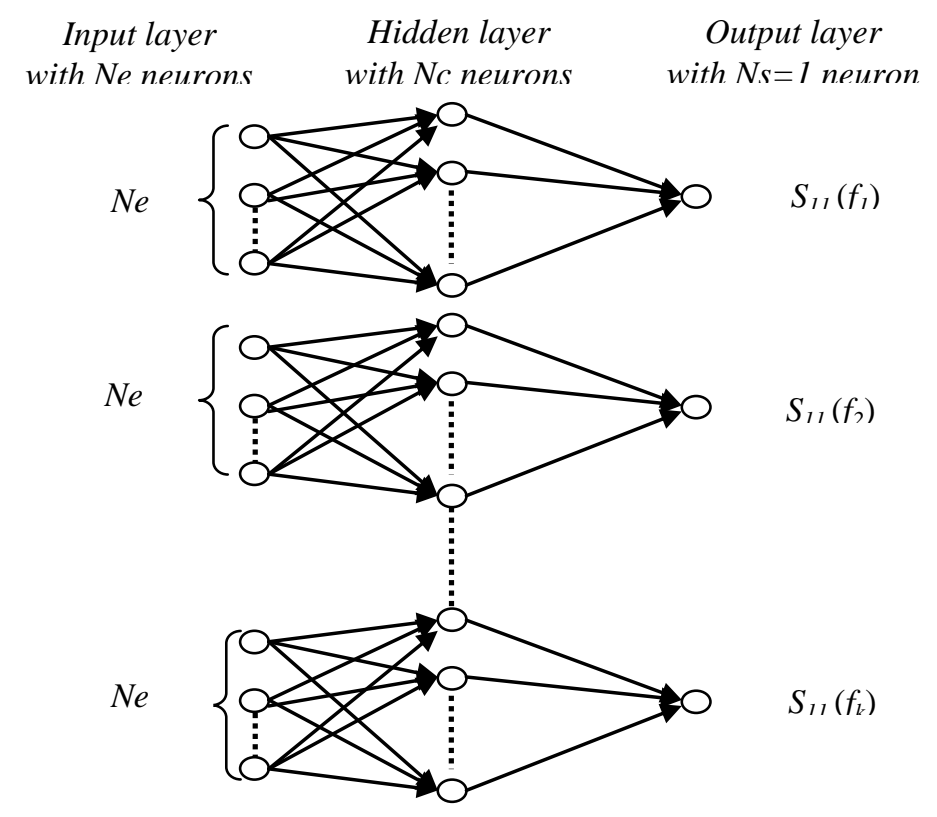

Fig. 1. Neural networks architecture.

\section{Social Spiders Optimization}

Erik Cuevas et al. [4] proposed a new swarm optimization algorithm called SocialSpiders Optimization (SSO). The (SSO) algorithm is based on the simulation of the cooperative behavior of social-spiders. A (SSO) process consists of two parts: members and communal web. The communal web is used as a mechanism to transmit in- 
formation among the colony members. This information is encoded as small vibrations depend on the weight and distance of the spider which has generated them, it is calculated according to:

$$
V i b_{i j}=W_{j} * \exp \left(-\left(d_{i j}\right)^{2}\right)
$$

Where $d_{i j}$ is the Euclidean distance between $i^{\text {th }}$ and $j^{\text {th }}$ spiders and $W_{j}$ indicates the weight of the $j^{\text {th }}$ spider dependent on the fitness value; is calculated by the following equation

$$
W_{i}=\frac{\text { Worst }-f\left(x_{i}\right)}{\text { Worst }- \text { Best }}
$$

Where $f\left(x_{i}\right)$ is the fitness value of the spider $\left(x_{i}\right)$. The values Best and Worst are defined as follows (considering a minimization problem):

$$
\text { Best }=\min _{i=1 \ldots N} f\left(x_{i}\right) \text { and Worst }=\max _{i=1 \ldots N} f\left(x_{i}\right)
$$

Every spider $\left(x_{i}\right)$ is able to consider three vibrations from other spiders as follows Fig. 2, Where $V i b_{c i}$ the nearest spider subject to having higher fitness, $V i b_{b i}$ the best spider in the swarm and $V i b_{f i}$ the nearest female spider.

The spiders members are divided by gender into two types: female spiders represent $65-90 \%$ of the entire population and the rest is the male spiders. They are calculated as follows:

$$
\begin{gathered}
N f=\text { floor }[(0.9-\operatorname{rand} * 0.25) * N] \\
N m=N-N f
\end{gathered}
$$

With $N, N_{f}, N m$ are the population, the number of females and the number of male respectively. (floor) rounds each element to the nearest integer, and rand is a random number in the unitary range $[0,1]$. A female spiders are updates its position as follows:

$$
\begin{aligned}
& X_{i}=X_{i}+\alpha \operatorname{Vib}_{c i}\left(X_{c}-X_{i}\right)+\beta \operatorname{Vib}_{b i}\left(X_{b}-X_{i}\right)+\delta(\text { rand }-0.5) \text { with probability } P F(6) \\
& X_{i}=X_{i}-\alpha \operatorname{Vib}_{c i}\left(X_{c}-X_{i}\right)-\beta \operatorname{Vib}_{b i}\left(X_{b}-X_{i}\right)+\delta(\text { rand }-0.5) \text { with probability 1-PF (7) }
\end{aligned}
$$

Where $\alpha, \beta, \delta$ and rand are random numbers between $[0,1]$ whereas $P F$ represents the threshold parameter. The individual $X_{c}$ and $X_{b}$ represent the nearest member to $X_{i}$ that holds a higher weight and the best individual of the entire population, respectively. 


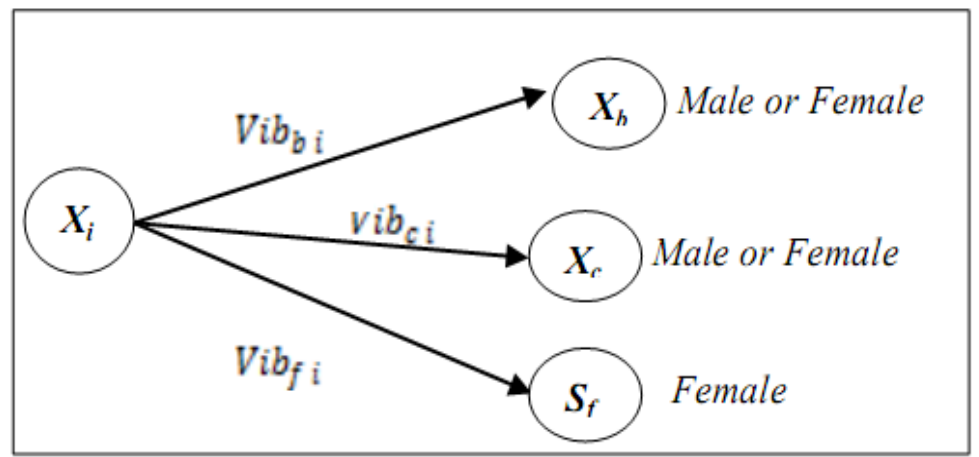

Fig. 2. Configuration of each special relation

The male population divided into two groups: Dominant members D with a weight value above the median value and non-dominant members ND with a weight value under the median value. The change of positions for the male spider can be modeled as follows:

$$
X_{i}=X_{i}+\alpha\left(\frac{\sum_{h=1}^{N_{m}} X_{h} W_{N_{f}+h}}{\sum_{h=1}^{N_{m}} W_{N_{f}+h}}-X_{i}\right) \text {, Male D }
$$

$$
X i=X i+\alpha \operatorname{Vibf} i(X f-X i)+\delta .(\text { rand }-0.5), \quad \text { Male ND }
$$

Where the individual $\left(X_{f}\right)$ represent the nearest female individual to the male member.

After all males and females spiders are update, the last operator is representing the mating behavior where only dominant males will participate with females who are within a certain radius called mating radius given by

$$
R=\frac{\sum_{d=1 \ldots n}\left(X_{d}^{h}-X_{d}^{l}\right)}{2 D}
$$

Where $X^{h}$ and $X^{l}$ are respectively the upper and lower bound for a given dimension and $n$ is the problem dimension.

Males and females which are under the mating radius generate new candidate spiders according to the roulette method. Each candidate spider is evaluated in the objective function and the result is tested against all the actual population members. If any member is worse than a new candidate, the new candidate will take the actual individual position assuming actual individual's gender. 


\section{Training Neural Networks using Social Spiders Optimization}

Training of neural networks is a complex task of great importance in the learning, where it is depends on adaptation of free network parameters, that is, on the proper selection of the neural weight values. The training of neural networks is to find an algorithm for optimized weights of networks to minimize the mean square error (MSE) described as follows

$$
M S E=\frac{1}{P T} * \sum_{P T} \sum_{N S}(Y S-Y)^{2}
$$

Where $P T$ the total number of training samples, $Y_{S}$ is the output of the network and $Y$ is the desired output.

$$
Y s=f_{2}\left(\sum_{N c} W s * f_{1}\left(\sum_{N e} W E * X\right)\right)
$$

With $f_{2}$ and $f_{1}$ are the activation functions (typically: sigmoid, tanh ...), $X$ is the input vector of NN.

Specialized learning algorithms are used for adaptation of these weight values. Among those algorithms, the most popular algorithm is a back-propagation method (BP) [5] based on a gradient descending. Lately, many populations based algorithms were proposed for training a neural network such as Particle Swarm Optimization (PSO) [6], Genetic Algorithms [7].

In this paper, the neural networks are trained by a SSO algorithm. The step-wise procedure for the implementation of SSO-NN can be summarized as follows:

Step.1. Define the neural network architecture (number of layers, number of neurons in each layer and the activation functions) and define the parameters of the SSO algorithm (number of population $N$, the number of female $\mathrm{N}_{\mathrm{f}}$, the number of male $\mathrm{N}_{\mathrm{m}}$, maximum iteration and the threshold $P F$ )

Step.2. Define the optimization parameters and the optimization problem:

- Design variables of the optimization problem: $W E$ and $W S$ the matrixes of input connection weights and output connection weights respectively. WE matrix of $N c$ rows and $\mathrm{Ne}$ columns and WS matrix of $N s$ rows and $N c$ columns.

- Define the optimization problem (fitness function): find the optimal WE and WS which minimizes the mean square error (MSE) equation (11).

Step.3. Initialize the population and evaluate the corresponding objective function value. For simplification, the population is decomposed into two groups, one represents the inputs weights population $W E p$ and the second one represents the out- 
put weights population WSp. Where, each group generates randomly, according to the population female, the population male and the number of neurons as follows:

$$
W E p=\operatorname{rand}(N * N c, N e), W S p=\operatorname{rand}(N s, N * N c)
$$

Step.4. Locate the best and the worst fitness, then calculate the weight of every spider in terms of its fitness equation (2)

Step.5. Move female spiders according to the female cooperative operator equations (6) and (7)

Step.6. Move male spiders according to the male cooperative operator equations (8) and (9)

Step.7. Perform the mating operation.

Step.8. If the stopping criteria are reached, the process is finished; otherwise, go back to Step 4.

\section{$4 \quad$ Application Examples and Application}

In this section, the ability of neural networks trained with SSO algorithm is assessed by applying it for the modeling of two microwave filters modeling test (Broad-band Eplane filters with improved stop-band [8] and H-plane waveguide filters considering rounded corners [9]) Fig. 3. The dimensions of the first filter and second filters are listed in Table 1.

The common parameters of each algorithms are chosen identical (population size $=30$ and number of iterations equal 10000), neural network parameters (Number of hidden neurons, input parameter and their limit, and frequency interval) of each structure are presented in Table 2. The activation functions are hyperbolic tangent function (Tansig), and linear function (Purelin) respectively. The other specific parameters of algorithms are given below.

PSO Settings: $c_{1}$ and $c_{2}$ are constant coefficients $c_{1}=c_{2}=2, w$ is the inertia weight decreased linearly from 0.9 to 0.2 .

SSO Settings: threshold parameter $P F=0.7$

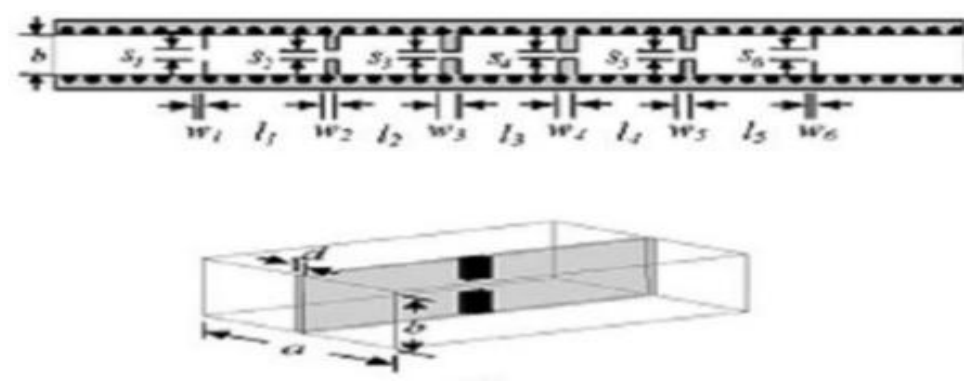

(a) 


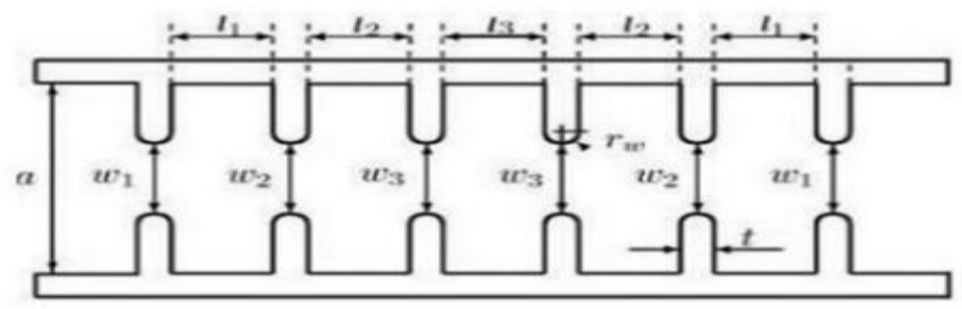

(b)

Fig. 3. (a) Broad-band e-plane filters with improved stop-band, (b) H-plane waveguide filters considering rounded corners

Table 1. The dimensions of the structures

\begin{tabular}{ccccc}
\hline Structure & \multicolumn{4}{c}{ Parameters and dimensions (mm) } \\
\hline & $\mathrm{a}$ & $\mathrm{b}$ & $\mathrm{w} 1=\mathrm{w} 6$ & $\mathrm{w} 2$ \\
& 7.112 & 3.556 & 0.254 & 0.889 \\
& $\mathrm{w} 3=\mathrm{w} 4$ & $\mathrm{~s} 1=\mathrm{s} 6$ & $\mathrm{~s} 2=\mathrm{s} 5$ & $\mathrm{~s} 3=\mathrm{s} 4$ \\
(a) & 1.219 & 1.092 & 0.610 & 0.508 \\
& $11=15$ & 12 & 13 & $\mathrm{~d}$ \\
& 7.697 & 6.452 & 6.706 & 0.25 \\
\hline & $\mathrm{a}$ & $\mathrm{b}$ & 11 & 12 \\
(b) & 34.85 & 15.79 & 22.88 & 25.57 \\
& 13 & $\mathrm{w} 1$ & $\mathrm{w} 2$ & $\mathrm{w} 3$ \\
& 25.57 & 15.10 & 9.03 & 7.98 \\
\hline
\end{tabular}

Table 2. Neural network parameters

\begin{tabular}{ccc}
\hline Structure & (a) & (b) \\
\hline $\mathrm{Nc}$ & 8 & 11 \\
& $0.20<\mathrm{W} 1<0.30$ & $13.95<\mathrm{W} 1<16.61$ \\
Input neurons & $0.71<\mathrm{W} 2<1.07$ & $8.13<\mathrm{W} 2<9.93$ \\
$\&$ interval & $0.97<\mathrm{W} 3<1.46$ & $7.182<\mathrm{W} 3<8.78$ \\
$(\mathrm{~mm})$ & $0.87<\mathrm{S} 1<1.31$ & \\
& $0.48<\mathrm{S} 2<0.74$ & \\
Frequency interval $(\mathrm{GHz})$ & {$[26-36]$} & {$[6.6-7.4]$} \\
$\mathrm{K}$ & 34 & 54 \\
\hline
\end{tabular}

Fig. 4 shows the convergence of SSO and PSO algorithms for minimize the MSE of neural networks for two filters above-mentioned. A comparison between the reflection coefficient responses obtained by using the SSO-NN, PSO-NN and the simulation are presented in Fig. 5. 
It is observed from Fig. 4 and Fig. 5 that, the SSO algorithms perform better in terms of convergence and approximation than the PSO algorithm.

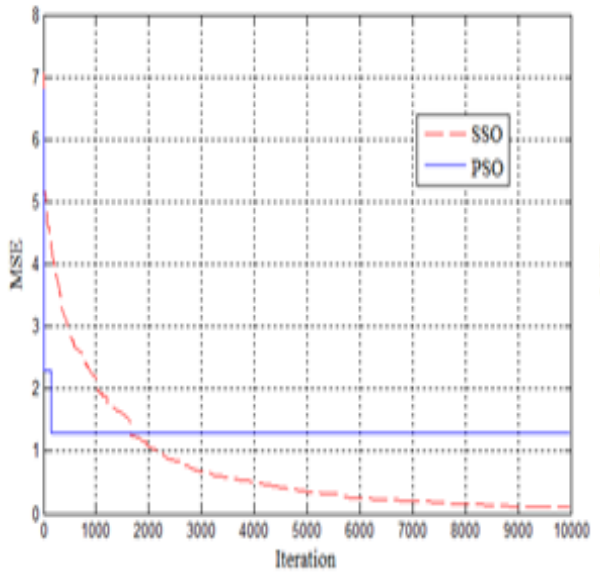

(a)

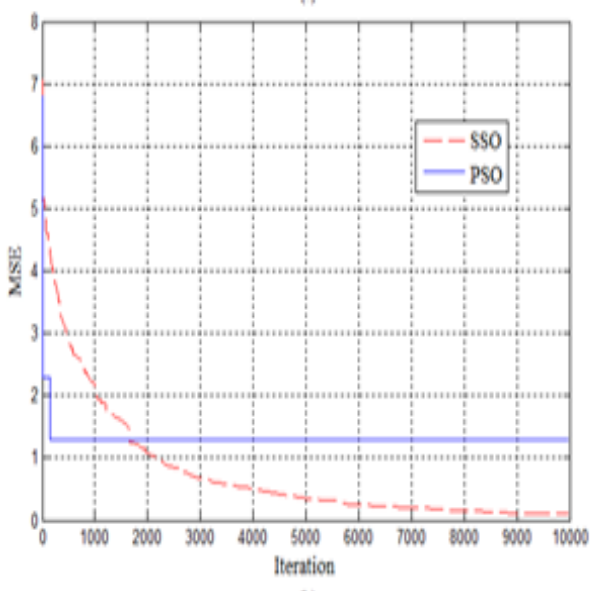

(b)

Fig. 4. Convergences of algorithms for minimize the MSE; (a) Broad-band e-plane filters with improved stop-band, (b) H-plane waveguide filters considering rounded corners.

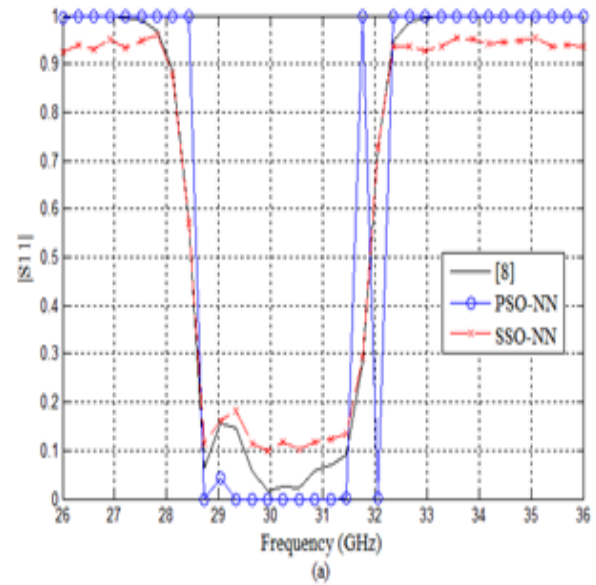

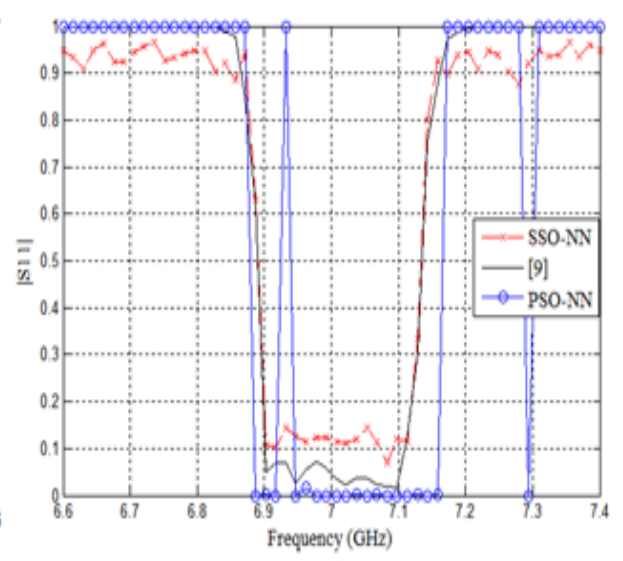

(b)

Fig. 5. The approximate reflection coefficient; (a) Broad-band e-plane filters with improved stop-band, (b) H-plane waveguide filters considering rounded corners. 


\section{Conclusion}

In the present work, we propose microwave filters (Broadband E-plane filters with improved stop-band and $\mathrm{H}$-plane waveguide filters considering rounded corners) modeling using neural networks trained by a social spider optimization (SSO) algorithm. For validated this neural network (SSO-NN) we compared them with those obtained using neural networks trained by PSO (PSO-NN). Excellent results of convergence and modeling are obtained.

\section{References}

1. Wang, Y., Yu, M., Kabir,H., Zhang, Q.J.: Application of Neural Networks in Space Mapping Optimization of Microwave Filters. International Journal of RF and Microwave Computer Aided Engineering, 22, 159-166 (2012).

2. Deshmukh, A. A., Kulkarni, S.D., Venkata, A.P.C., Phatak, N.V.: Artificial Neural Network Model for Suspended Rectangular Microstrip Antennas. Procedia Computer Science, 49, 332-339 (2015).Author, F., Author, S., Author, T.: Book title. 2nd edn. Publisher, Location (1999).

3. Sivia, J. S., Pharwaha, A. P. S., Kamal, T. S.: Analysis and design of circular fractal antenna using artificial neural networks. Progress in Electromagnetics Research B, 56, 251267 (2013).

4. Cuevas, E., Cienfuegos, M.: A new algorithm inspired in the behavior of the social-spider for constrained optimization. Expert Systems with Applications, 41, 412-425(2014).

5. Jwo, D.J., Chin, K.P.: Applying Back-propagation Neural Networks to GDOP Approximation,. The Journal of Navigation, 55, 97-108 (2002).

6. Gyanesh, D., Prasant, K. P., Sasmita, K. P.: Artificial Neural Network Trained by Particle Swarm Optimization for Non-Linear Channel Equalization. Expert Systems with Applications, 41, 3491-3496 (2014).

7. Ding, S., Zhang, Y., Chen, J., Weikuan, J.: Research on Using Genetic Algorithms to Optimize Elman Neural Networks. Neural Computing and Applications, 23, 293-297 (2013).

8. Xu, Z., Guo, J., Qian, Ch., Dou, W.: Broad-Band E-plane Filters with Improved StopBand Performance. IEEE Microwave and Wireless Components Letters, 21(7), 350-752 (2011).

9. Diaz Caballero, E., Morro, J., Belenguer, A., Esteban, H., Boria, V.: CAD Technique for Designing H-Plane Waveguide Filters Considering Rounded Corners. IEEE MTT-S International Microwave Symposium Digest, pp. 1-3, WA, USA (2013). 